\title{
The Application of Modified Artificial Neural Network on Tensile Behaviour of 316L Austenitic Stainless Steel
}

\author{
Jian Peng ${ }^{1,2}$, Jian Peng ${ }^{1,2, a}$ and Kaishang $\mathrm{Li}^{1,2}$ \\ 1 School of Mechanical Engineering Changzhou University, Changzhou, 213164, China. \\ 2 Jiangsu Key Laboratory of Green Process Equipment, Changzhou University, Changzhou, 213164, China.
}

\begin{abstract}
In this paper, genetic algorithm (GA) was used to enhance the accuracy of back propagation artificial neural network (ANN) for the tensile behaviour of 316L. With GA optimization, the random weight and threshold between different layers in original ANN model can be updated constantly to describe the characteristics of $316 \mathrm{~L}$ austenitic stainless steel accurately, just considering the nodes of hidden layer. According to the sample distribution, the data were divided into training and test. When the model was set to predict test data, the results showed coincident with the experimental ones, and errors of the data between prediction and experiment displayed the small values at room and intermediate temperatures.
\end{abstract}

\section{Introduction}

Constitutive models are usually treated as the mathematical description, which can reflect the macroscopic characteristics of materials. Conventional models are able to obtain the results, but can hardly calculate them efficiently and accurately, so the calculation of parameters will cost a lot of time.

In recent years, with the development of artificial intelligence, machine learning is becoming mainstream gradually. As one of the most popular methods of machine learning, artificial neural network was used widely in many fields [1], due to the simplified calculation, large scope of application and high accuracy. When the mechanical properties of many engineering materials were involved, the artificial neural network showed high practicability, which was superior to the models based on mathematical theories and physical phenomenon. In this regard, it was applied in flow stress prediction of metallic materials, such as $\mathrm{Nb}-\mathrm{Ti}$ alloy steel [2], stainless steel [3], $\mathrm{Cu}-\mathrm{Al}$ [4] and $\mathrm{Al}-\mathrm{Cu}-$ $\mathrm{Mg}-\mathrm{Pb}$ alloys [5]. Especially the back propagation artificial neural network (BP ANN), it seems like the brain of human beings, which can reduce errors by adjusting the weight and threshold autonomously. But the weight and threshold might fall into local optimal values easily without algorithm optimization.

Genetic Algorithm (GA) is considered as an optimized algorithm, which was studied by many researchers, including mechanical property [6], structural design [7] and reliability analysis [8]. With GA, the BP ANN constitutive model can be established to describe the tensile flow behaviour of 316L austenitic stainless steel accurately, and only a small amount of work should be accomplished, when the artificial neural network is considered as a constitutive model of $316 \mathrm{~L}$ austenitic stainless steel.

\footnotetext{
${ }^{\mathrm{a}}$ Corresponding author : joepengjian@163.com
} 


\section{Material and Experiment}

From Fig.1 (a), 316L austenitic stainless steel was chosen as the material, and tensile experiment was executed on the tensile machine, which showed in Fig.1 (b).

According to the tensile experiments at different temperatures and strain rates, the relationship between flow stress and strain can be found, but the key point is how to quantitate the qualitative relation.

(a)
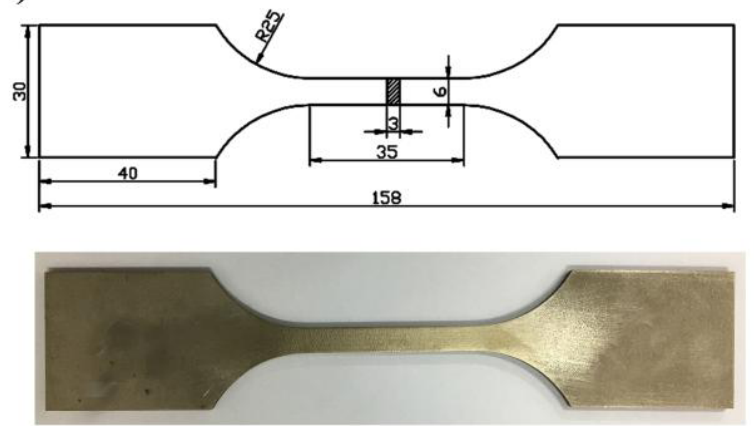

(b)

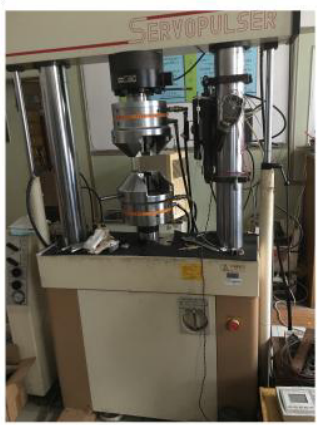

Figure.1 (a) Structure of 316L austenitic stainless steel and (b) tensile machine

\section{Constitutive model}

\subsection{Artificial neural network}

Back propagation (BP) neural network is one of the artificial neural networks, which imitates the biological brain learning, and the system of dealing with information consists of a lot of neurons. According to Fig.2, it takes the single hidden layer as an example. If the number of nodes in the input layer is $m$, and the number of nodes in the output layer is $n$. then the $m$ dimensional to $n$ dimensional mapping can be realized by the BP neural network of a single hidden layer in any cases [9]. In addition, the selection of node number in the hidden layer can affect the performance of neural network, the little nodes lead to the low ability of identifying sample, while too many nodes lead to more training time. The number of nodes in the hidden layer can not be determined by a regular way, but the range of nodes can be estimated with an empirical formula shown in Eq.1.

$$
l=\sqrt{m+n}+a
$$

Where $a$ represents the adjustment parameter range from 1 to 10 , and $l, m, n$ represent the range of nodes in hidden layer, input layer and output layer respectively.

The procedure of BP neural network can be divided into two parts. One is the forward transmission of information, and the other one is the backward transmission of errors. In the forward transmission of information, the current output can be obtained from the output of the former layer, the weight between current and former layer, the threshold of former layer, as well as the transfer function. However, in the latter part, which means the backward transmission of error, the error will reduce by changing the weight between input layer and hidden layer, as well as the weight between hidden layer and output layer, corresponding to the change of threshold in hidden and output layer. 
(a)

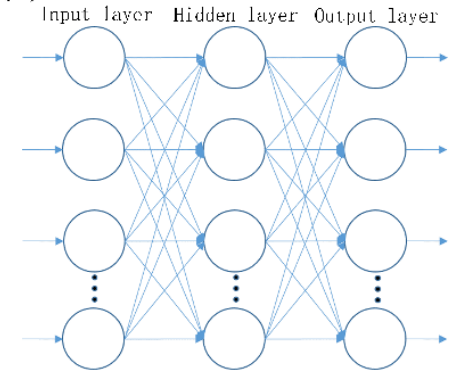

(b)

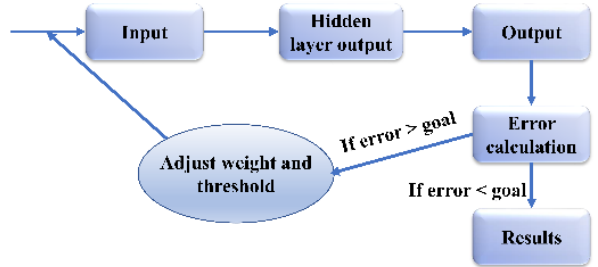

Figure 2. (a) Structure and (b) the procedure of back propagation artificial neural network

\subsection{Genetic Algorithm (GA)}

GA is one of the intelligence algorithm based on evolution, and the problem of local optimum can be solved efficiently with it. According to some rules about biology, including heredity, mutation and so on, the procedure of GA shows as Fig.3. According to Fig.3, it is found that, the first step is to code the original weight and threshold like biological gene code. Then the error between prediction and expectation data is considered as fitness function. The last step consists of selection, cross and mutation, in order to simulate the biological evolution.

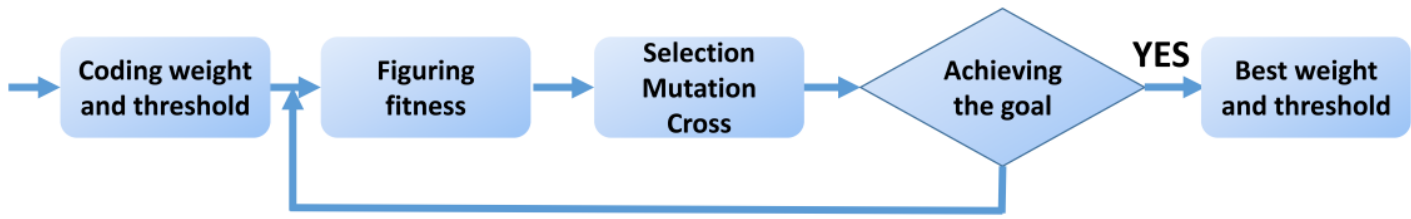

NO

Figure.3 Procedure of genetic algorithm

\section{Application of constitutive model}

According to the theories above, the application of BP artificial neural network can predict the flow behaviour of $316 \mathrm{~L}$ austenitic stainless steel, including the prediction of flow stress at room and intermediate temperatures. It is determined that input layer has 3 neurons (strain, temperature and strain rate), while the output layer has 1 neuron (flow stress). Depending on the formula shown in Eq.1, the number of hidden layer nodes can be estimated with the range from 3 to 12 .

\subsection{Normalization of sample}

Due to the difference of strain rate, temperature and strain, the convergence rate and accuracy of the network can be influenced. Thus, the dealing of normalization is shown in Eq.2, and the normalized data can focus on 0.1-0.9.

$$
x_{n}=0.1+0.8 * \frac{x-x_{\min }}{x_{\max }-x_{\min }}
$$

Where, $x$ is the original input and output data, $x_{n}$ is the data of normalized data, $x_{\text {min }}, x_{\max }$ are the maximum and minimum of one set sample, such as strain rate.

\subsection{Training and test}


The data at different temperatures and strain rates need to be divided into training data and test data. Table 1 gives the distribution of training data and test data, three strain rates $(1 \mathrm{e}-2 / \mathrm{s}, 5 \mathrm{e}-3 / \mathrm{s}, 1 \mathrm{e}-3 / \mathrm{s})$ were selected as test data at three temperatures $(373 \mathrm{~K}, 473 \mathrm{~K}, 573 \mathrm{~K})$ respectively, and the rest are training data.

Table. 1 Distribution of sample data

\begin{tabular}{|c|c|c|c|c|}
\hline & $293 \mathrm{~K}$ & $373 \mathrm{~K}$ & $473 \mathrm{~K}$ & $573 \mathrm{~K}$ \\
\hline $5 \mathrm{e}-4 / \mathrm{s}$ & train & train & train & train \\
\hline $1 \mathrm{e}-3 / \mathrm{s}$ & train & train & train & test \\
\hline $5 \mathrm{e}-3 / \mathrm{s}$ & train & train & test & train \\
\hline $1 \mathrm{e}-2 / \mathrm{s}$ & train & test & train & train \\
\hline
\end{tabular}

According to the training of some sample data, the error is minimal when the number of nodes in hidden layer is eight. Training parameters are shown in Table 2. Function 1 and function 2 represent activation function of hidden layer and output layer, and function 3 represents the training function of network. Figure 4(a) gives the comparison between experimental and prediction of training data. And Figure 4(b) gives the comparison between experimental and prediction data of test.

Table. 2 Training parameters of artificial neural network

\begin{tabular}{|c|c|c|c|c|c|}
\hline Function 1 & Function 2 & Function3 & Epochs & Target error & Learning rate \\
\hline Sigmoid (tansig) & Linear (purelin) & Lm (trainlm) & 1000 & $1 \mathrm{e}-5$ & 0.01 \\
\hline
\end{tabular}

(a)

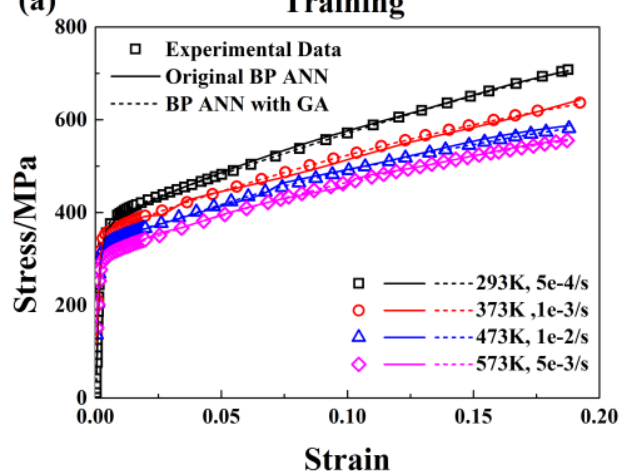

(b)

Test

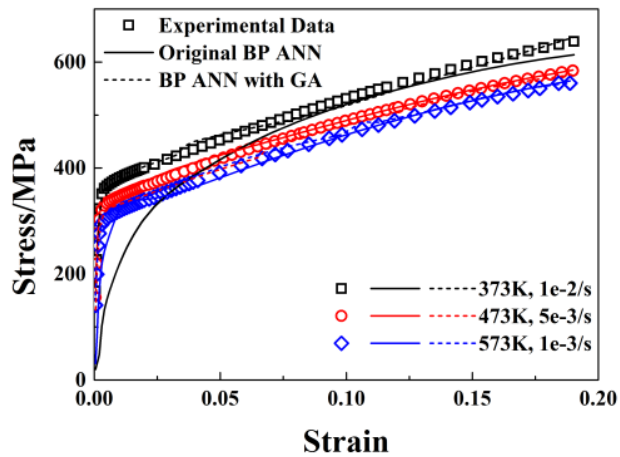

Figure. 4 Comparison between experimental and prediction data by (a) training and (b) test

Correlations and errors at the temperatures and strain rates are shown in Table 3. Among the results, it can be seen that correlation coefficients are all at high level, and the average errors of training data and test data are all at low level, which indicates the rationality of network training, and the prediction accuracy is well.

Table.3 Correlation coefficients and average errors at different temperatures and strain rates

\begin{tabular}{|c|c|c|c|c|c|}
\hline Temperature (K) & $\begin{array}{l}\text { Strain } \\
\text { rate(/s) }\end{array}$ & $\begin{array}{l}\text { Correlation } \\
\text { coefficients }\end{array}$ & $\begin{array}{c}\text { Average } \\
\text { errors }\end{array}$ & $\begin{array}{l}\text { Correlation } \\
\text { coefficients }\end{array}$ & $\begin{array}{c}\text { Average } \\
\text { errors }\end{array}$ \\
\hline & & \multicolumn{2}{|c|}{ Original BP ANN } & \multicolumn{2}{|c|}{ BP ANN with GA } \\
\hline 293 (Training) & $5 e-4$ & 0.998 & 3.29 & 0.999 & 2.21 \\
\hline 373 (Training) & 1e-3 & 0.995 & 2.94 & 0.998 & 1.13 \\
\hline 473 (Test) & 5e-3 & 0.993 & 4.26 & 0.999 & 1.53 \\
\hline 573 (Training) & 1e-2 & 0.998 & 1.15 & 0.999 & 0.29 \\
\hline
\end{tabular}




\section{Conclusion}

(1) A constitutive model based on BP artificial neural network and genetic algorithm was established, which can describe the flow behavior of $316 \mathrm{~L}$ austenitic stainless steel at room and intermediate temperatures properly.

(2) According to the comparison between experimental data and prediction, not only the training data can get high accuracy, but the test data can be found in good performance by BP artificial neural network.

(3) When comparing the results of original BP artificial neural network with those of BP artificial neural network with genetic algorithm, it can be found that the prediction accuracy of the improved model is higher than that of original model.

\section{Acknowledgements}

This project is supported by National Natural Science Foundation of China (Grant No. 51505041) and Natural Science Foundation of the Jiangsu Higher Education Institutions of China (Grant No. 16KJB460002).

\section{References}

1. P. Li, L.K. Zeng and A.Z. Rui, Comp. Appl. Software, 150, 184 (2008).

2. S. W. Wu, X. G. Zhou, G. M. Cao, Z. Y. Liu, G. D. Wang, Mat. Des, 15, 2 (2017).

3. A. R. Sayyed, A. Fakhreddin, F. Saghar, Mater. Sci. Eng. A, 15, 10 (2016).

4. M. Amirjan, H. Khorsand, M. H. Siadati, R. E. Farsani, J. Mater. Res. Technol, 4, 2 (2013).

5. H. R. Rezaei Ashtiani, P. Shahsavari, J. Alloy. Compd, 5, 12 (2016).

6. G. Petronea, V. Meruaneb, Compos Part A: Appl. Sci. Manufac, 94, 3 (2017).

7. L. Wang, A. Koliosa, T. Nishinoa, P. L Delafina, T. Birdb, Compos. Struct, 153, 10 (2016).

8. M. Shahnewaza, R. Machialb, M. S. Alamc, A. Rteilc, Eng. Struct, 107, 1 (2016).

9. W.D. Zeng, Y. Shu and Y.G. Zhou, Rare. Metal. Mat. Eng, 10, 33(2004). 